\title{
Journal of Diabetes and Obesity
}

\section{Clinical Outcomes in Patients with Diabetes Mellitus Using a Medically Supervised Commercial Weight Reduction Program Compared to Standard Care in an Endocrine Specialty Clinic}

\author{
R.C. Kennedy ${ }^{*}$, A. Inankur ${ }^{1}$, A. Qureshi ${ }^{1}$, K.J, McQuerry ${ }^{2}$, L.R. Reynolds ${ }^{1}$ \\ ${ }^{1}$ Division of Endocrinology and Metabolism, University of Kentucky, Lexington, KY \\ ${ }^{2}$ Department of Statistics, University of Kentucky
}

*Corresponding author: R.C. Kennedy, Division of Endocrinology and Metabolism, University of Kentucky, Lexington, KY, E-mail: rckennedy@live.com

\begin{abstract}
Aims: To evaluate metabolic and anthropometric changes achieved and maintained by diabetic patients (pts) in a structured weight loss (WL) program vs usual care in an endocrine clinic (UCG).

Materials and Methods: This retrospective comparison study examined 38 diabetic pts with BMI $>25$ undergoing the active weight loss phase of WL determined by patient goal weight and then followed for 6 months in weight maintenance phase (WM). Multiple endpoints were assessed at baseline including BMI and hemoglobin A1c (A1c). Endpoints were reassessed at 6 months in WM. The usual care group (UCG) was obtained through chart review of 26 diabetic pts with BMI $>25$ in an endocrine specialty clinic who completed an education program including lifestyle counseling by a certified diabetic educator. Data were analyzed using ANCOVA and protected LSD, adjusting for age, gender, and baseline weight.

Results: Patients showed a change in BMI at 6 months of $-6.8 \pm 0$ (bsl $44 \pm 8.4$ ) and $-0.7 \pm 1.1$ (bsl $35 \pm 6.2$ ) for HMR ${ }^{\circledR}$ pts and UCG, respectively $(\mathrm{p}<0.05)$. HMR $®$ pts had $13.4 \% \pm 3 \%$ WL $v s 7.9 \% \pm 4 \%$ in UCG p $=0.34) .6$ month A1c was similar in HMR pts $(7.5 \% \pm 2$; bsl $8.3 \pm 1.9)$ and UCG $(7.5 \% \pm 2.3$; bsl $9.8 \pm 1.8)$. HMR ${ }^{\circledR}$ pts had a reduction of total medication usage of $28 \%$, with at least one medication discontinued in $80 \%$ of pts, while the UCG had only $23 \%$ of pts with discontinuation of at least one medication $(\mathrm{p}<0.05)$.

Conclusions: Compared to CDE-led diabetic education emphasizing lifestyle change, pts in an intensive WL program utilizing weekly coaching, meal replacements, and exercise, had a significant decrease in BMI and achieved a similar A1c with reduction in medication requirements.
\end{abstract}

Received date: March 28, 2016

Accepted date: September 20, 2016

Published date: September 23, 2016

Citation: Kennedy, R.C., et al. Clinical Outcomes in Patients with Diabetes Mellitus Using a Medically Supervised Commercial Weight Reduction Program Compared to Standard Care in an Endocrine Specialty Clinic. (2016) J Diab Obes 3(2): 51- 56.

DOI: $10.15436 / 2376-0494.16 .838$

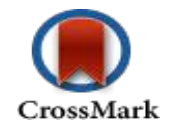

\section{Introduction}

A recent assessment of the 2005 U.S. National Ambulatory Medical Care Survey (NAMCS) indicated that a significant proportion of U.S. ambulatory patients with Type 2 diabetes mellitus present with obesity and greater clinical acuity than patients with diabetes alone ${ }^{[1]}$. This rising prevalence of obesity in diabetic patients has significantly contributed to the complexity of medical management due to the subsequent comorbidities, and most healthcare providers struggle with this aspect of care in their diabetic patients. This difficulty in achieving and maintaining weight loss has led to the evaluation of various non-surgical and surgical approaches to weight loss in Type 2 diabetic patients ${ }^{[2,3]}$.

Copyrights: (C) 2016 Kennedy, R.C. This is an Open access article distributed under the terms of Creative Commons Attribution 4.0 International License. 
According to the American Diabetes Association's (ADA's) 2015 consensus guidelines, there is sufficient evidence to support weight loss for all overweight or obese individuals who have or are at risk for Type 2 diabetes as modest weight loss has been shown to reduce insulin resistance and usually results in improved glycemic control. The ADA guidelines suggest that non-surgical approaches to weight loss including a variety of differently composed macronutrient diets which promote energy-restricted eating patterns, combined with physical activity and behavior modification, may be effective in achieving clinically significant weight loss. They further promote that intensive lifestyle programs with frequent follow up are often required to achieve significant reductions excess weight ${ }^{[4]}$.

Less clear are the associated health risks of obesity in patients with Type 1 diabetes. Although both the UK Prospective Diabetes Study (UKPDS) and Diabetes Control and Complications Trial (DCCT) interventions proved an overall reduction in cardiovascular risk, the subset of Type 1 diabetic patients with the highest quartile of weight gain had associated worsening of cardiovascular risk factors suggesting the potential harm of obesity in Type 1 diabetes patients ${ }^{[5]}$. Further, given that these patients tend to have greater insulin sensitivity then type 2 diabetic patients, caloric reduction in this group can be more difficult to implement without increased hypoglycemia. There have been no studies to our knowledge assessing nonsurgical weight loss interventions in patients with Type 1 diabetes and the associated benefits of weight loss in this group.

Obesity, in populations with and without diabetes, has been associated with increased cardiovascular morbidity and mortality ${ }^{[6]}$. Modest weight loss $(5-10 \mathrm{~kg})$ is shown to reduce cardiovascular risk factors including impaired glucose tolerance, total cholesterol and LDL cholesterol levels ${ }^{[7,8]}$. In bariatric surgery patients, greater degrees of sustained weight loss (10 - 40 $\mathrm{kg}$ ) are associated with risk factor improvement over 10 years. A reduction in cardiovascular events after bariatric surgery, however, is not always correlated with weight loss. This lack of correlation between weight loss and cardiovascular events suggests the need to explore weight loss independent of effects of bariatric surgery ${ }^{[9]}$. Toward this end, patients in commercial weight reduction programs provide a unique opportunity to study the metabolic effects of substantial weight loss without the potential confounding of bariatric surgery.

Based on the recommendations by the ADA, we intended to evaluate the efficacy of a highly structured weight loss program in diabetic patients compared to standard outpatient management of weight loss in similar patients. Our current outpatient strategy for assisting patients with weight loss includes counseling and informative literature from diabetic educators and physicians regarding diet and exercise during routine office visits. Health Management Resources (HMR) is a commercial weight reduction program, described best in U.S. News And World report ${ }^{[10]}$, affiliated with University of Kentucky in which patients receive structured weight loss plans consisting of low-calorie meal replacements, physical activity recommendations, and weekly behavioral modification education classes with medical supervision of complex patients such as those with diabetes through attainment of goal weight(Phase I). This is followed by a recommended minimum of 18 months of maintenance behavioral modification classes with continued supplemental low calorie meal replacements (Phase II). The purpose of this study was to evaluate the metabolic and anthropometric changes achieved and maintained using the HMR program versus usual care, including lifestyle counseling by certified diabetes educators, in the University of Kentucky Endocrine Specialty Clinic, for obese patients with type 1 or 2 diabetes.

\section{Materials and Methods}

This was a retrospective comparison study assessing for weight loss, glycemic control, metabolic changes, anthropometric changes, and maintenance of weight loss in patients with diabetes participating in a highly structured weight loss program versus a standard, usual care outpatient approach. Patients for this study were recruited from those existing patients followed in the HMR program affiliated with the University of Kentucky ${ }^{[10]}$. The usual care group consisted of diabetic patients treated for weight loss as part of their routine care at the Endocrine Specialty Clinic, University of Kentucky, who received lifestyle education in a AADE certified education program led by a certified diabetes educator (CDE). Patients were recruited from October 5, 2010 to March 4, 2013.

HMR patients recruited to participate in the study were at least 18 years of age; had an established diagnosis of Type 1 or Type 2 diabetes; had completed at least 12 weeks of PHASE I to obtain predetermined weight loss goal before entering PHASE II; and had a baseline BMI $>25$. After obtaining informed consent, study patients' medical charts were accessed for: 1) Phase 1 Pre-program labs (Hemoglobin A1c, complete metabolic panel (CMP), and lipid panel), anthropometric data, blood pressure and medical history; as well as medication list 2) In PHASE II (maintenance) peripheral blood samples evaluating for hgb A1c, lipid profile, and complete metabolic panel were than drawn at 6 months with reassessment of anthropometric data and medication list. Throughout study, patients maintained weekly meetings with reinforcement of exercise and dietary recommendations, and continued use of meal replacements as desired.

The usual care group of subjects, who had initiation of lifestyle education by a certified diabetes educator, was randomly selected for chart review. Patients were at least 18 years of age, had an established diagnosis of either Type 1 or Type 2 diabetes, and were overweight or obese based on body mass index. Study patients' medical charts were accessed for medical history, baseline anthropometric characteristics, blood pressure, medication list, and baseline labs at their first visit to the Endocrine Clinic in the Kentucky Clinic. Medical charts were than further assessed for data at 6 months after their initial visit for changes in anthropometric characteristics, blood pressure, hemoglobin A1C, and medications.

HMR patients' pre-program laboratory data was assessed through review of the patient medical chart. All labs drawn during or prior to PHASE I, were under the supervision of the HMR staff or the patient's primary care provider. Additional peripheral blood samples were obtained using standard phlebotomy techniques by designated research staff 6 months after initiation of PHASE II at the Lexington, KY HMR office. The amount of blood drawn at each visit was approximately 20 milliliters. Anthropometric data was collected at each follow up appointment.

Insulin cost analysis was calculated from prescribed insulin units for a 30 day period, with monetary values based on information from Drugs.com on October 2, 2014. Based on 
that information, approximation of cost for analog insulin was 200.00 dollars per 10 milliliter vial; Regular and NPH insulin was 25.00 dollars per 10 milliliter vial; and U-500 insulin was 1200.00 dollars per 20 milliliter vial.

\section{Statistical analysis}

Data were analyzed using ANCOVA and protected LSD, adjusting for age, gender, and baseline weight to allow for baseline differences in group. All analyses were complete using PROC GLM, SAS 9.4 (SAS Institute Inc., Cary, NC, USA.). Results are expressed as least squares means \pm standard error.

\section{Results}

\section{Subjects and baseline characteristics}

A total of 38 diabetic patients in the HMR program were enrolled in the trial. Overall, $23 \%(n=9)$ of the participants were lost to follow up without reported adverse effects. Only 2 patients in each group were classified as Type 1 diabetics and so further effort to separate them in analysis was not made. A retrospective chart review was performed consisting of 26 randomly selected diabetic patients who underwent weekly lifestyle education classes for weight loss by a certified Diabetic educator (control group), as part of their routine care at the Endocrine Clinic at the University of Kentucky. Of the HMR patients, 34 $\%$ of the patients who completed the study were on insulin at baseline $(n=10)$ compared to $73 \%$ of the control group $(n=19)$. Weight, BMI, Hemoglobin a1c, and medication list including insulin use was assessed for patients prior to initiation of care at the clinic, as well as 6 and 12 months after participation in classes had begun.

Baseline characteristics between the two groups including age, gender, anthropometry, duration of diabetes, and hemoglobin A1C are demonstrated in Table 1. Significant differences were noted between the 2 groups including a higher baseline age, weight, BMI, duration of diabetes, as well as a lower $\mathrm{HbA1c}$ in the HMR group compared to the Control group.

Table 1:

\begin{tabular}{|l|c|c|}
\hline \multicolumn{1}{|c|}{ HRM } & Control \\
\hline Age & $58( \pm 2.0)$ & $50( \pm 2.1)$ \\
\hline Duration of Diabetes Year & $14( \pm 10.4)$ & $11.94( \pm 10.0)$ \\
\hline Mean weight lbs & $298( \pm 10.6)$ & $223( \pm 6.3)$ \\
\hline BMI & $44( \pm 1.5)$ & $35( \pm 1.0)$ \\
\hline A1C & $8.3( \pm 0.3)$ & $9.8( \pm 0.3)$ \\
\hline Female & 8 & 12 \\
\hline Male & 21 & 14 \\
\hline
\end{tabular}

\section{Data comparison from intervention period}

Evaluation of the HMR Group from initiation of HMR program to 6 months post maintenance was compared to the control group. Examination of anthropometric measurements showed that the HMR group was noted to have a decrease in BMI of $6.82 \pm 1.00$. This was statistically significant when compared to the control group who had an average decrease in of $0.70 \pm 1.12(\mathrm{p}<0.05)$ over the 6 month period of evaluation (Figure 1). Although not statistically significant, this corresponded to a $13.4 \% \pm 3 \%$ percent weight loss on average in the HMR group versus only $7.9 \% \pm 4 \%$ in the control Group $(\mathrm{p}=$
0.34) (Figure 2). For HMR participants, mean waist circumference at baseline was 55 inches and after 6 months, this decreased to 47 inches. Waist circumference measurements were not routinely performed in the endocrine specialty clinic and were not available on chart review of the usual care group.

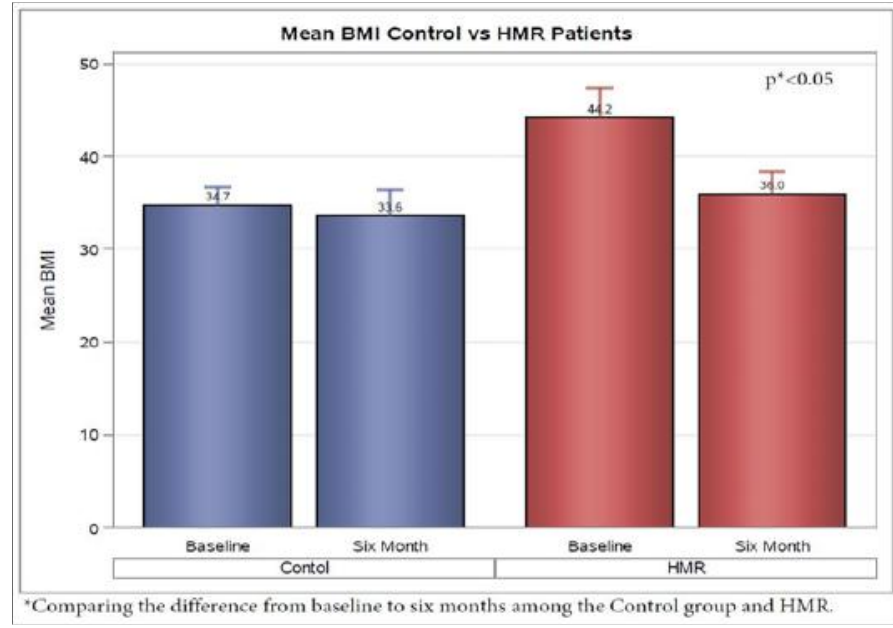

Figure 1: HMR participants decreased BMI $6.82 \pm 1.00$ on average from baseline to 6 months; the control group decrease was $0.70 \pm 1.12$ $(\mathrm{p}<0.05)$.

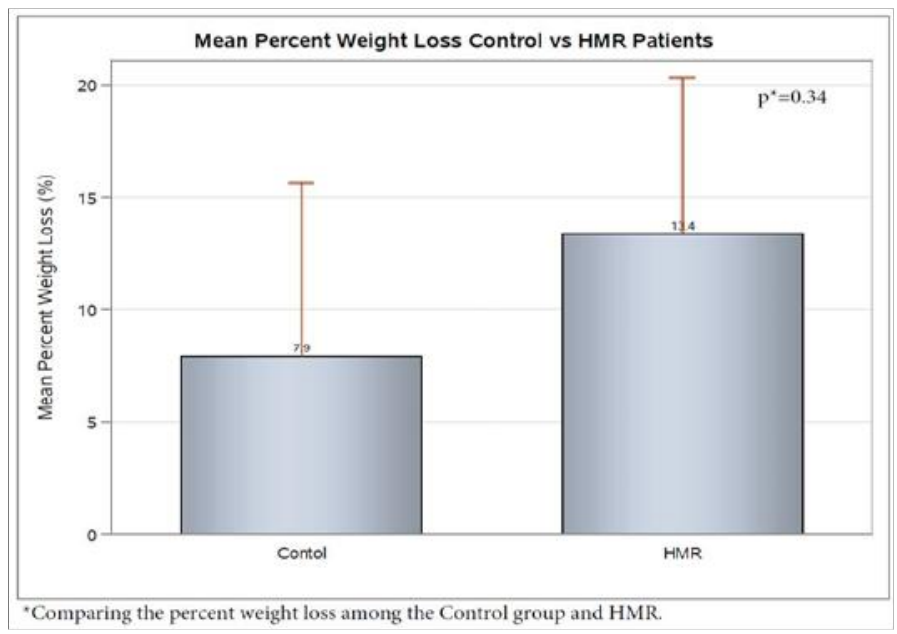

Figure 2: Mean weight loss was $13.4 \% \pm 3 \%$ percent in HMR group weight loss on average versus only $7.9 \% \pm 4 \%$ in control $(p=0.34)$

Evaluation of change in hemoglobin A1C from baseline to the end of the study was evaluated revealed that both groups demonstrated a significant decrease with end average A1C being similar at $7.5 \% \pm 2.0$ in the HMR group and $7.5 \%$ $\pm 2.3 \%$ in the control group. The overall mean $\mathrm{A} 1 \mathrm{C}$ decrease in the HMR participants was 0.8 while, the control group was 2.3 over 6 months. When adjusted for age, gender, and bsl weight, the average A1C decreased in the HMR participants was $0.58 \pm$ 0.38 ; while, the control group was $2.31 \pm 0.42(\mathrm{p}<0.05)$ (Figure 3). 


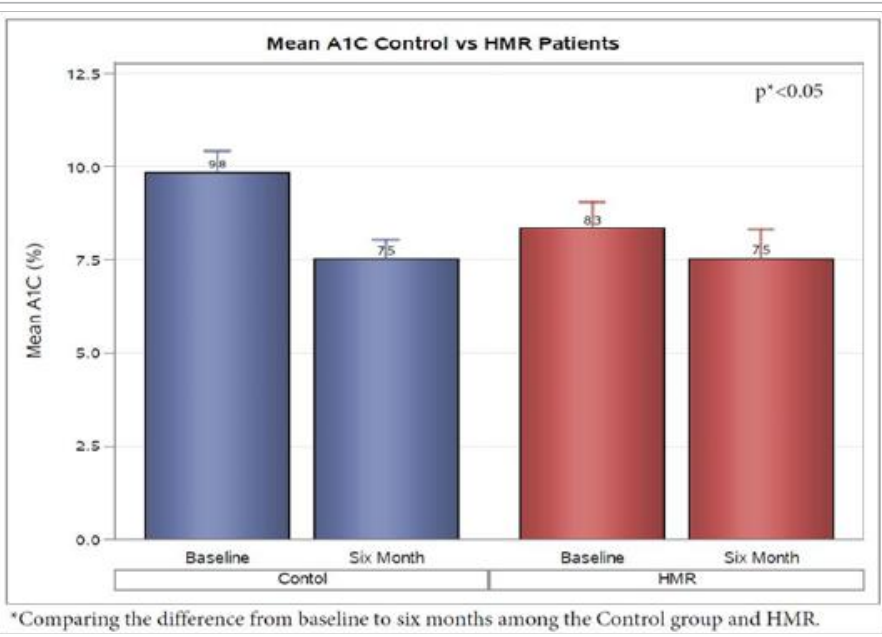

Figure 3: The overall mean A1C decrease in the HMR participants was 0.8 while, the control group was 2.3 over 6 months. When adjusted for age , gender, and bsl weight, the average A1C decreased in the HMR participants was $0.58 \pm 0.38$; while, the control group was $2.31 \pm 0.42$ $(\mathrm{p}<0.05)$. Hemoglobin a1c achieved at 6 months was similar in HMR pts $(7.5 \% \pm 2.0)$ and Control group $(7.5 \% \pm 2.3)$.

Comparison of the changes in the participants medication list showed a reduced average number of daily medications needed in the HMR group by a total of two, while the control group had a slight increase over the 6 month period $(\mathrm{p}<0.05)$. This equated to reduction of total medication usage of $28 \%$ in HMR ${ }^{\circledR}$ pts, with at least one medication discontinued in $80 \%$ of pts, while the control had only $23 \%$ of pts with discontinuation of at least one medication $(\mathrm{p}<0.05)$ (Figure 4$)$.

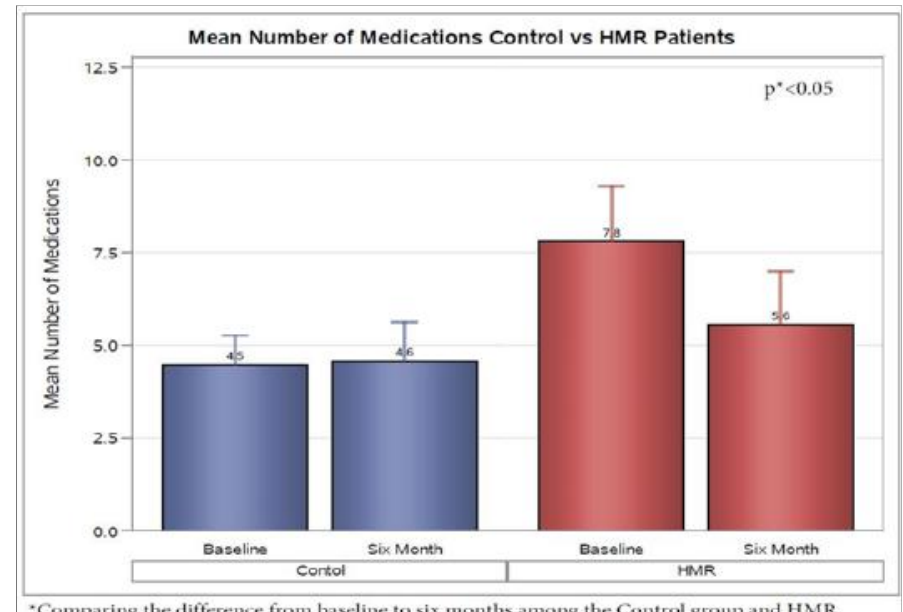

"Comparing the difference from baseline to six months among the Control group and HMR.

Figure 4: On average, HMR participants reduced the average number of overall medications needed by 2 while the control group had a slight increase over the 6 month period $(\mathrm{p}<0.05)$. This equated to reduction of total medication usage of $28 \%$ in $\mathrm{HMR} \otimes$ pts, with at least one medication discontinued in $80 \%$ of pts, while the control had only $23 \%$ of pts with discontinuation of at least one medication $(p<0.05)$

Analysis of insulin use demonstrated a decrease in HMR patients of $32.88 \pm 12.63$ units per day and an increase of $18.88 \pm 9.16$ units per day in the control group from baseline to 6 month follow up $(\mathrm{p}<0.05)$ (Figure 5). As expected, the average 30 day cost of insulin from baseline to 6 months for HMR participants decreased $\$ 197.28 \pm \$ 106.41$, but the control group increased $\$ 131.91 \pm \$ 77.20(\mathrm{p}<0.05)$ (Figure 6). No significant change was noted from initiation to finish in the two groups baseline lipid profile or metabolic panel.

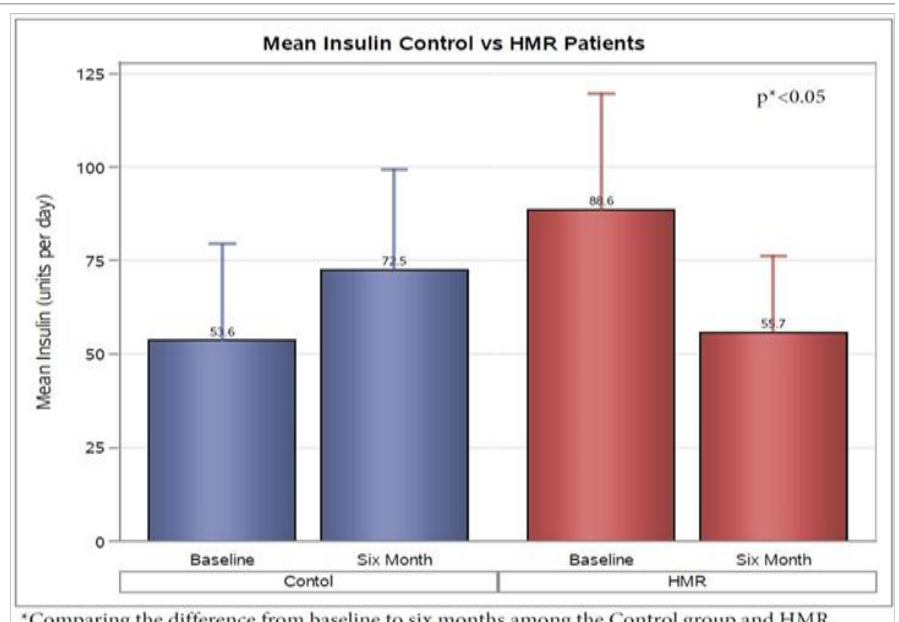

"Comparing the difference from baseline to six months among the Control group and HMR.

Figure 5: Among insulin users in the study (HMR patients $\mathrm{n}=10$; Control $\mathrm{n}=19)$, average daily scheduled insulin use decreased $32.88 \pm$ 12.63 units in HMR patients and increased $18.88 \pm 9.16(\mathrm{p}<0.05)$ units in the control group from baseline to 6 month follow up.

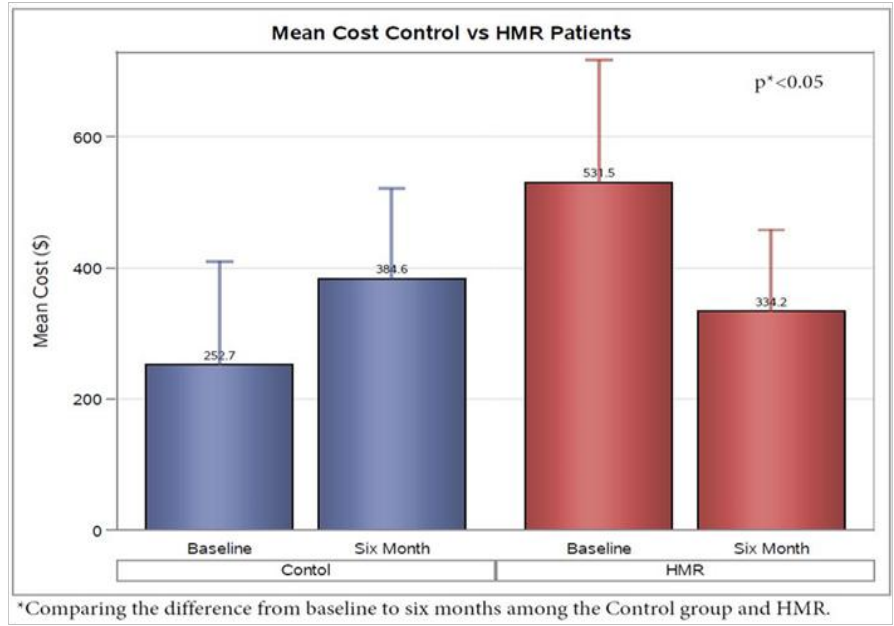

Figure 6: As expected, the average 30 day cost of insulin from baseline to 6 months for HMR participants decreased $\$ 197.28 \pm \$ 106.41$, but the control group increased $\$ 131.91 \pm \$ 77.20(\mathrm{p}<0.05)$. 30 day Cost analysis was calculated from scheduled insulin units used based on information from Drugs.com with approximation of analog insulin's at $200 \$$ per $10 \mathrm{ml}$ vial; Regular and NPH insulin at $25 \$$ per $10 \mathrm{ml}$ vial; $\mathrm{U}-500$ insulin at $1200 \$$ per $20 \mathrm{ml}$ vial.

\section{Discussion}

Evidence for the benefits of weight loss and exercise in individuals with high risk for the development of Type 2 Diabetes has been shown in multiple studies ${ }^{[1-17]}$. This includes the Diabetes Prevention Program, in which those individuals attaining at least 150 minutes of physical activity per week and a loss of seven percent of body weight had a $58 \%$ reduced incidence of T2DM when compared to control group over 2.8 years ${ }^{[1]}$. Currently The American Diabetes Association and European Association currently recommend that highly motivated patients with A $1 \mathrm{c}<7.5 \%$ at diagnosis, should be given the opportunity to engage in lifestyle changes for 3 to 6 months before embarking on pharmacotherapy ${ }^{[18]}$. However, data on the effectiveness in lifestyle interventions in those already diagnosed with T2DM is less robust, but it has been demonstrated that lifestyle intervention with effective weight loss and increased aerobic capacity can lead to increased insulin sensitivity and potential remission 
in those recently diagnosed ${ }^{[19]}$. The Look AHEAD trial has additionally been shown to have significant reductions alc, lipids, and medication burden through implementation of intensive lifestyle interventions ${ }^{[20]}$.

In our study we looked at two groups managed in both a specialty medical practice setting and structured behavioral weight loss program. In regards to absolute weight loss, statistical significance was not different when looking at baseline to endpoint, though the HMR group's BMI reduction was significantly more than that of the control group. While the weight reduction was not impressively different, the effect of the programs on medication usage was significant as we saw an overall reduction in the HMR group versus an increase in the group managed in a traditional endocrine practice. Further analysis of subjects prescribed insulin demonstrated an increase in insulin dosing in the control group as opposed to a decrease insulin dose in the HMR group. This translated to a significant cost savings from baseline in the HMR group with equivalent hgbalc achieved.

Certainly initial glycemic control being worse in the usual care group may have necessitated overall increase in insulin usage within that group. Another contributor to the difference may have been a result of the emphasis of lifestyle and behavioral changes in the structured weight loss program as opposed to pharmacotherapy as seen in traditional medical clinics. While the consequences of hyerinsulinemia remain controversial, there have been multiple studies linking elevated insulin levels to atherosclerosis, Tumor growth, and hypertension ${ }^{[21-26]}$. When looking at exogenous replacement of insulin, a study by S.E. Holden et al., demonstrated a dose dependent relationship of increased all-cause mortality, major cardiovascular events, incidence of hypoglycemia in patients taking $>0.6$ units $/ \mathrm{kg}$, and incidence of cancer ${ }^{[27]}$. Insulin use also contributes to the economic burden of Type 2 DM in the United States, which was noted to be 245 billion dollars in 2012. When compared to 2007, this was estimated to be a $41 \%$ increase ${ }^{[28]}$. To this end, placing patients with chronic medical conditions which correlate to obesity, such as T2DM, in programs which demonstrate the potential to diminish medication burden should be strongly considered in regards to long term morbidity and mortality, as well as diminished economic impact.

Obesity complicated by T2DM is a condition affected by multiple variables and consideration should be given to the management of diet, physical activity, behavioral modification, as well as pharmacotherapy. Structured weight loss programs often provide focus on elements beyond pharmacotherapy which may be difficult to effectively manipulate in a traditional medical practice. Unfortunately, due to a multitude of factors, including insurance coverage and physician time constraints, the majority of patients are not offered nor pursue behavioral weight loss programs as a part of their diabetic treatment plan ${ }^{[29]}$. When compared to hypocaloric balanced diets, structured programs such as HMR consisting of low energy diets, which are diets consisting of $<1200 \mathrm{kcal} / \mathrm{day}$, combined with modest amounts of exercise have been shown to be more effective in both initial weight loss and maintenance over a 5 year follow up period ${ }^{[30]}$. In our study, this approach demonstrated not only a decrease in weight, but also improvement in glucose control with reduction in medication burden which may be the true beneficial significance in major lifestyle interventions compared to traditional management. Therefore, consideration should be given to struc- tured behavioral weight loss programs as part of the initial treatment for type 2 diabetes mellitus in association with obesity.

In summary, the data from our study suggests that when compared to CDE-led diabetic education emphasizing lifestyle change for weight loss, diabetic patients in an intensive lifestyle program utilizing weekly behavioral coaching, meal replacements and exercise, had a significant decrease in BMI and achieved a similar A1c with greater reduction in medication use. In particular, HMR diabetic patients utilizing insulin had significant reduction in dose and cost from baseline compared to an overall increase in patients managed in a traditional endocrinology specialty clinic.

Acknowledgments: This study was funded in part by HMR (health management resources) and was presented at the Annual Obesity Society Meeting in 2014.

\section{References}

1. Neumiller, J.J., Sclar, D.A., Robison, L.M., et al. Rate of obesity in U.S. ambulatory patients with diabetes mellitus: a national assessment of office-based physician visits. (2009) Prim Care Diabetes 3(3): 197199.

2. Wing, R.R., Wadden, T.A., Espeland, M. Surgical vs behavioral therapy for weight loss in patients with type 2 diabetes. (2008) JAMA 299(18): 2146-2147.

3. Pi-Sunyer, X., Blackburn, G., Brancati, F.L., et al. Reduction in weight and cardiovascular disease risk factors in individuals with type 2 diabetes: one-year results of the look AHEAD trial. (2007) Diabetes Care 30(6): 1374-1383.

4. Standards of medical care in diabetes--2015: summary of revisions. (2015) Diabetes Care 38 Suppl: S4.

5. Bloomgarden, Z.T. Prevention of obesity and diabetes. (2003) Diabetes Care 26(11): 3172-3178.

6. Whitlock, G., Lewington, S., Sherliker, P., et al. Body-mass index and cause-specific mortality in 900000 adults: collaborative analyses of 57 prospective studies. (2009) Lancet 373(9669): 1083-1096.

7. Poobalan, A., Aucott, L., Smith, W.C., et al. Effects of weight loss in overweight/obese individuals and long-term lipid outcomes--a systematic review. (2004) Obes Rev 5(1): 43-50.

8. Torgerson, J.S., Hauptman, J., Boldrin, M.N., et al. XENical in the prevention of diabetes in obese subjects (XENDOS) study: a randomized study of orlistat as an adjunct to lifestyle changes for the prevention of type 2 diabetes in obese patients. (2004) Diabetes Care 27(1): 155-161.

9. Sjostrom, L., Peltonen, M., Jacobson, P., et al. Bariatric surgery and long-term cardiovascular events. (2012) JAMA 307(1): 56-65.

10. http://health.usnews.com/best-diet/hmr-diet

11. Knowler, W.C., Barrett-Connor, E., Fowler, S.E., et al. Reduction in the incidence of type 2 diabetes with lifestyle intervention or metformin. (2002) N Engl J Med 346(6): 393-403.

12. Tuomilehto, J., Lindström, J., Eriksson, J.G., et al. Prevention of type 2 diabetes mellitus by changes in lifestyle among subjects with impaired glucose tolerance. (2001) N Engl J Med 344(18): 1343-1350. 13. Crandall, J.P., Knowler, W.C., Kahn, S.E., et al. The prevention of type 2 diabetes. (2008) Nat Clin Pract Endocrinol Metab 4(7): 382-393. 14. Ramachandran, A., Snehalatha, C., Mary, S., et al. The Indian Diabetes Prevention Programme shows that lifestyle modification and metformin prevent type 2 diabetes in Asian Indian subjects with impaired glucose tolerance (IDPP-1). (2006) Diabetologia 49(2): 289-297.

15. Saito, T., Watanabe, M., Nishida, J., et al. Lifestyle modification and prevention of type 2 diabetes in overweight Japanese with impaired fasting glucose levels: a randomized controlled trial. (2011) Arch Intern Med 171(15): 1352-1360. 
16. Knowler, W.C., Fowler, S.E., Hamman, R.F., et al. 10-year follow-up of diabetes incidence and weight loss in the Diabetes Prevention Program Outcomes Study. (2009) Lancet 374(9702): 1677-1686.

17. Aucott, L., Poobalan, A., Smith, W.C., et al. Weight loss in obese diabetic and non-diabetic individuals and long-term diabetes outcomes--a systematic review. (2004) Diabetes Obes Metab 6(2): 85-94.

18. Nathan, D.M., Buse, J.B., Davidson, M.B., et al. Medical management of hyperglycemia in type 2 diabetes: a consensus algorithm for the initiation and adjustment of therapy: a consensus statement of the American Diabetes Association and the European Association for the Study of Diabetes. (2009) Diabetes Care 32(1): 193-203.

19. Ades, P.A., Savage, P.D., Marney, A.M., et al. Remission of Recently Diagnosed Type 2 Diabetes Mellitus With Weight Loss and Exercise. (2015) J Cardiopulm Rehabil Prev 35(3): 193-197.

20. Pi-Sunyer, X., Blackburn, G., Brancati, F.L., et al. Reduction in weight and cardiovascular disease risk factors in individuals with type 2 diabetes: one-year results of the Look AHEAD trial. (2007) Diab Care 30(6): 1374-1383.

21. Muis, M.J., Bots, M.L., Bilo, H.J., et al. High cumulative insulin exposure: a risk factor of atherosclerosis in type 1 diabetes? (2005) Atherosclerosis 181(1): 185-192.

22. Muis, M.J., Bots, M.L., Grobbee, D.E., et al. Insulin treatment and cardiovascular disease; friend or foe? A point of view. (2005) Diabet Med 22(2): 118-126.
23. Cao, W., Ning, J., Yang, X., et al. Excess exposure to insulin is the primary cause of insulin resistance and its associated atherosclerosis. (2011) Curr Mol Pharmacol 4(3): 154-166.

24. Gallagher, E.J., LeRoith, D. The proliferating role of insulin and insulin-like growth factors in cancer. (2010) Trends Endocrinol Metab 21(10): 610-618.

25. Soleimani, M. Insulin resistance and hypertension: new insights. (2015) Kidney Int 87(3): 497-499.

26. Horita, S., Seki, G., Yamada, H., et al. Insulin resistance, obesity, hypertension, and renal sodium transport. (2011) Int J Hypertens 2011: 391762.

27. Holden, S.E., Jenkins-Jones, S., Morgan, C.L., et al. Glucose-lowering with exogenous insulin monotherapy in type 2 diabetes: dose association with all-cause mortality, cardiovascular events and cancer. (2015) Diabetes Obes Metab 17(4): 350-362.

28. Economic costs of diabetes in the U.S. in 2012. (2013) Diabetes Care 36(4): 1033-1046.

29. Ades, P.A. A lifestyle program of exercise and weight loss is effective in preventing and treating type 2 diabetes mellitus: Why are programs not more available? (2015) Prev Med 80: 50-52.

30. Anderson, J.W., Konz, E.C., Frederich, R.C., et al. Long-term weight-loss maintenance: a meta-analysis of US studies. (2001) Am J Clin Nutr 74(5): 579-584.
Ommega Online Publishers

Journal Title: Journal of Diabetes and Obesity (JDO)

Journal Short Name: J diabetes Obes
Journal ISSN: 2356-0494

E-mail: diabetes@ommegaonline.com

Website: www.ommegaonline.org 